\title{
Complicación post-resección endoscópica de espesor completo en paciente octogenario
}

Anna Pigem Rodeja ${ }^{1}$

Pere Planellas Giné

Ramón Farrés Coll ${ }^{1}$

David Busquets Casals²

1 Unidad de Cirugía Colorrectal del Servicio de Cirugía General. Hospital

Universitario Dr. Josep Trueta de Girona.

${ }^{2}$ Unidad de Endoscopia del Servicio del Aparato Digestivo. Hospital

Universitario Dr. Josep Trueta de Girona.

\section{CASO CLÍNICO.}

Presentamos el caso de un paciente de 87 años sin alergias medicamentosas conocidas y con antecedentes de HTA, flutter auricular, cardiopatía con marcapasos implantado hace 20 años, insuficiencia mitral moderada-severa con hipertensión pulmonar moderada secundaria, insuficiencia renal crónica y con cirugías previas de colecistectomía laparoscópica y faquectomía bilateral. Sigue tratamiento habitual con Sintrom ${ }^{\circledR}$, ácido acetilsalicílico, enalapril y bisoprolol. Es remitido a la consulta de Coloproctología por presentar anemia ferropénica con test de sangre oculta en heces positiva. El paciente describe astenia sin limitación de sus actividades diarias y sin otra sintomatología añadida. La exploración física es normal y el tacto rectal no muestra lesiones evidentes. La analítica general destaca $\mathrm{Hb} 9.3 \mathrm{~g} / \mathrm{dL}$, creatinina $1.6 \mathrm{mg} / \mathrm{dl}$ y marcadores tumorales dentro del rango de la normalidad. La colonoscopia muestra una úlcera de $2 \mathrm{~cm}$ de aspecto infiltrativo localizada en colon ascendente y diverticulosis severa de sigma (fig.1). 


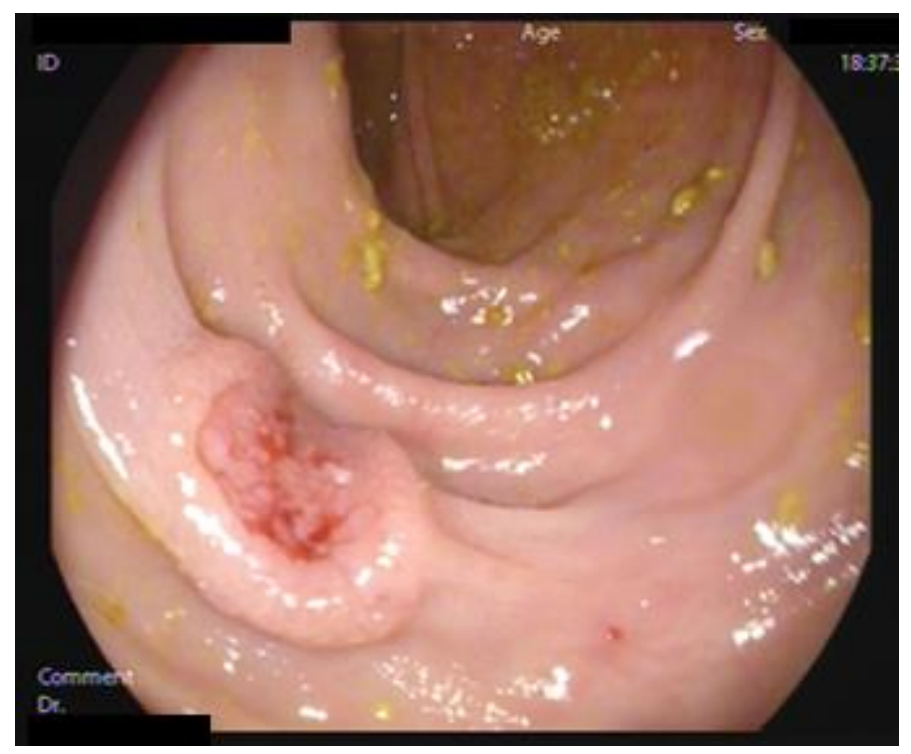

Figura 1. Lesión ulcerada visualizada durante la colonoscopia.

El caso es presentado en comité multidisciplinar que acuerda realizar una nueva colonoscopia con intención resectiva. La colonoscopia describe una lesión plana en colon ascendente, con centro deprimido ulcerado (Paris 0-IIc) de 18-20 mm, de aspecto tumoral NICE 3. Se procede a marcaje de márgenes con soft coagulación y resección transmural de la lesión con kit FTRD (endoscopic full-thickness resection, Ovesco®). La introducción de la lesión dentro del capuchón del kit resulta compleja por la probable infiltración tumoral de la misma. Durante la liberación del clip Ovesco® se produce una laceración completa de pared luminal, quedando el clip Ovesco $₫$ adherido a uno de los márgenes de la resección. Se procede a intento de cierre del defecto con varios clips, no siendo técnicamente posible. Ante la perforación de colon post-resección endoscópica se propone cirugía urgente.

A la valoración, el paciente se encuentra estable hemodinámicamente, con saturación 98\%, presentando distensión abdominal con leve dolor a la palpación de fosa ilíaca derecha y sin signos de irritación peritoneal. Se decide intervención quirúrgica laparoscópica, encontrándose líquido libre seroso en parietocólico, hiperemia de asas de intestino delgado y perforación de $2 \mathrm{~cm}$ en colon ascendente (fig.2). 


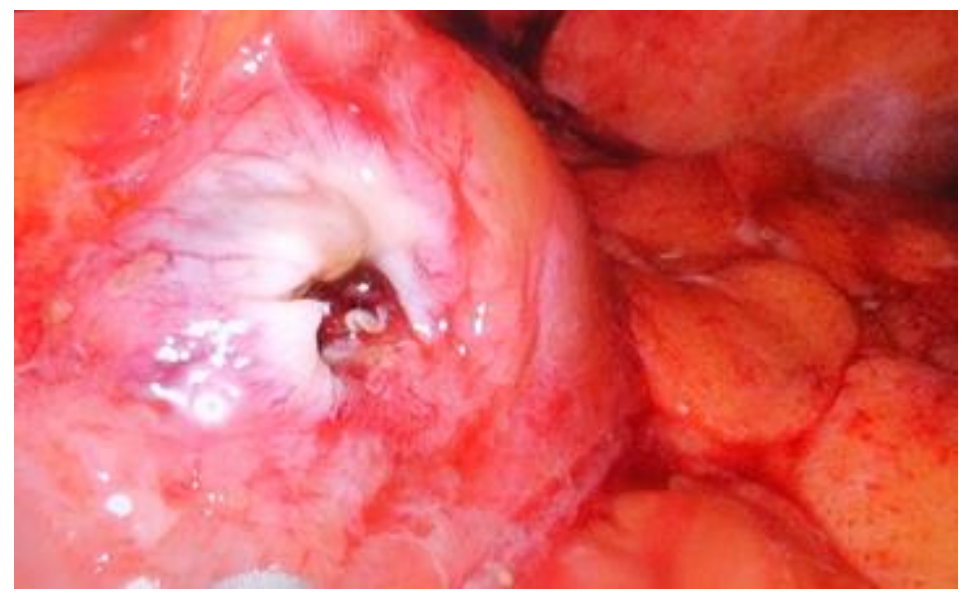

Figura 2. Perforación post-resección de espesor completo observada durante la cirugía.

Se procede a realización de hemicolectomía derecha laparoscópica reglada con anastomosis intracorpórea íleo-transversa latero-lateral mecánica con cierre del ojal con sutura continua.

En el tercer día postoperatorio el paciente presenta hipotensión i rectorragias abundantes, por lo que se realiza revisión endoscópica urgente que muestra hemorragia activa (jet) en un punto de la anastomosis. Se consigue hemostasia con colocación de 2 clips y esclerosis con inyección de adrenalina $(1 \mathrm{ml})$. El resto del postoperatorio cursa sin incidencias y el paciente es dado de alta al séptimo día.

La anatomía patológica mostró un adenocarcinoma de ciego de 3,5 × 2,2 cm, PT1N0(0/22)MO, con invasión tumoral submucosa de 2500 micras, sin invasión linfática, vascular o perineural y con márgenes libres de tumor (R0) (fig.3). 


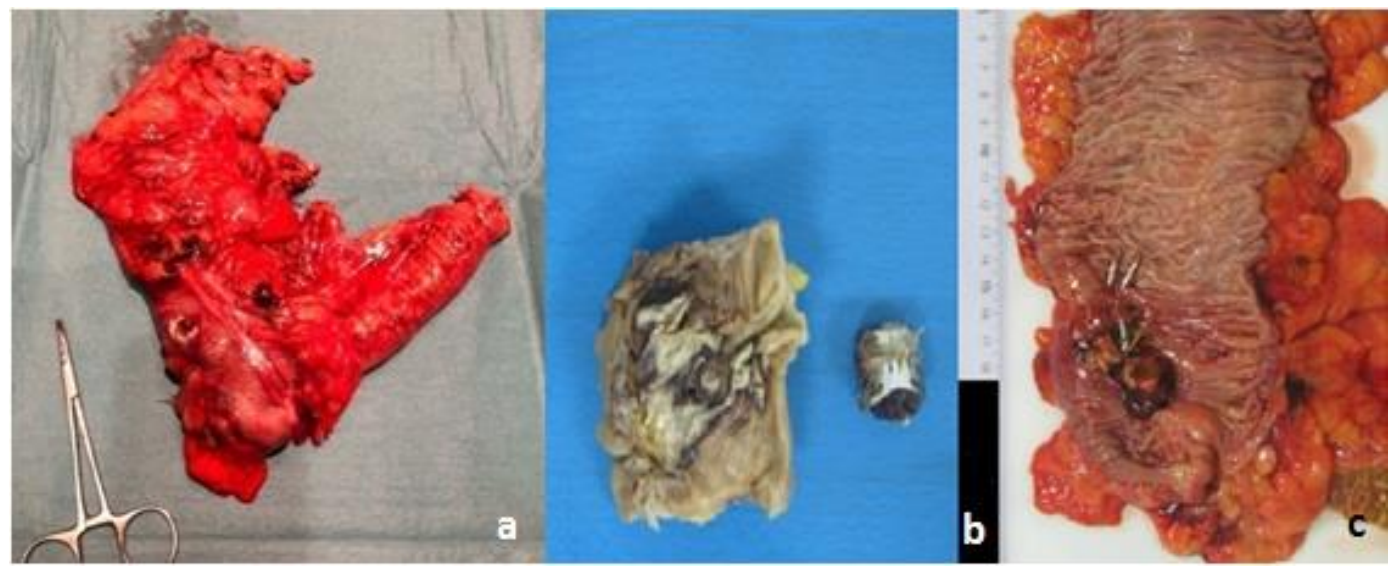

Figura 3. Pieza quirúrgica con la perforación (a). Corte de colon ascendente con la lesión tumoral y clip Ovesco® (b). Corte de colon con los clips en intento de cierre (c).

\section{DISCUSIÓN}

Con el aumento de la esperanza de vida y una población octogenaria cada vez más presente en los servicios de cirugía, existe una discusión permanente sobre si la edad avanzada puede suponer un límite por sí misma en la toma de decisiones quirúrgicas.

Un tercio de la mortalidad precoz en estos pacientes se debe a la limitación del esfuerzo terapéutico ante el fracaso del tratamiento ya establecido o ante eventos adversos del mismo ${ }^{1}$.

Los avances terapéuticos actuales permiten resecciones endoscópicas más amplias y profundas en el colon. La aparición de dispositivos endoscópicos como el sistema OTSC (over the scope clip, Ovesco Endoscopy GMBH, Tübingen, Germany), permiten tratar, de una forma rápida y segura, los efectos adversos (perforación u hemorragia) durante el mismo procedimiento, evitando así su manejo quirúrgico².

La resección endoscópica de espesor completo (EFTR) es una nueva técnica que expande el espectro de métodos endoscópicos de resección para carcinomas mucosos, lesiones submucosas y lesiones que no muestran lifting sign (elevación tras inyección submucosa). Es un tratamiento curativo actualmente aceptado para la neoplasia intraepitelial no invasiva y el cáncer colorrectal que afecta la capa más superficial de la submucosa (<1000 micras) y que presenta criterios histológicos de buen pronóstico. Puede 
ser una alternativa paliativa en pacientes muy seleccionados en los que el riesgo quirúrgico es muy elevado, como los pacientes ancianos con comorbilidades importantes ${ }^{3}$.

Entre las complicaciones más frecuentes se encuentra la perforación colónica y el sangrado de la serosa. La principal limitación de la EFTR es el tamaño de la resección, dado que aumenta el riesgo de resección R1 y la dificultad del cierre ${ }^{4}$. En casos seleccionados, las perforaciones de $<1 \mathrm{~cm}$ pueden resolverse mediante clips. La principal indicación del sistema Ovesco® han sido las perforaciones iatrogénicas de 1 a $3 \mathrm{~cm}$ durante endoscopias diagnósticas o terapéuticas, no recomendándose resecciones de lesiones mayores de $30 \mathrm{~mm}$, ya que el diámetro del capuchón es de $21 \mathrm{~mm}$. La tasa de éxito del cierre es del $91 \%$.

Ante el fracaso del Ovesco $®$, debe plantearse el tratamiento quirúrgico urgente. El abordaje y la técnica quirúrgica dependen de la situación clínica del paciente y la experiencia del cirujano ${ }^{6}$.

En los pacientes octogenarios sometidos a estas actuaciones, la deliberación de las opciones terapéuticas y los objetivos a conseguir debe hacerse de forma precoz y por parte de todo el equipo médico, el paciente y la familia ${ }^{7}$.

\section{BIBLIOGRAFIA}

1. Glance, L. G., Osler, T. M., Neuman, M. D. (2014). Redesigning Surgical Decision Making for High-Risk Patients. N Engl J Med.2014;370(15), 1379-1381.

2. Albrecht, H., Raithel, M., Braun, A., Nagel, A., Stegmaier, A., Utpatel, K., et al. Endoscopic full-thickness resection (EFTR) in the lower gastrointestinal tract. Tech Coloproctol. 2019;23(10):957-963.

3. Bartell, N., Bittner, K., Kaul V., Kothari, T. H., Kothari, S. Clinical efficacy of the over-the-scope clip device: A systematic review. World J Gastroenterol 2020;26(24):3495-3516. 
4. Marín-Gabriel, J.C., Díaz-Tasede, J., Rodríguez-Muñoz, S., del-Pozo-García, A. J., Ibarrola-Andrés, C. Colonic endoscopic full-thickness resection (EFTR) with the over-the scope device (FTRD): a short case series. Rev Esp Enferm Dig. 2017;109:230-233.

5. Junquera, F., Martínez-Bauer, E., Miquel, M., Fort, M., Gallach, M., Brullet, E., et al (2011). OVESCO: un sistema prometedor de cierre endoscópico de las perforaciones del tracto digestivo. Gastroenterol Hepatol.2011;34(8):568-572.

6. Donatelli, G., Cereatti, F., Dhumane, P., Vergeau, B. M., Tuszynski, T., Marie, C., et al. Closure of gastrointestinal defects with Ovesco clip: long-term results and clinical implications. Ther Adv Gastroenterol. 2016; 9(5):713-721.

7. Lavanchy, J. L., Holzgang, M. M., Haltmeier, T., Candinas, D., Schnüriger, B. Outcomes of emergency abdominal surgery in octogenarians: A single-center analysis. Am J Surg. 2019; 218:248-254. 